\title{
Identification of hepatosensitive region and their neural connections in the hippocampus of rats
}

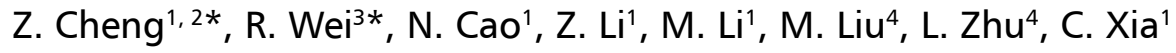 \\ 'Department of Human Anatomy, Basic Medical College of Guilin Medical University, China \\ ${ }^{2}$ Department of Anatomy, Basic Medical Department of Henan Vocational College of Nursing, China \\ ${ }^{3}$ College of Biotechnology, Guilin Medical University, China \\ ${ }^{4}$ Affiliated Hospital of Guilin Medical University, China
}

[Received: 24 December 2020; Accepted: 1 February 2021; Early publication date: 23 February 2021]

Background: Visceral function localisation of the brain is very complex. For many years, people have been actively exploring the neural mechanism regulating visceral and substance metabolism, clarifying the complex relationship between the brain and peripheral nervous system related to the regulation of visceral activity, and analysing its complex neural pathways. The brain is the advanced centre of visceral function regulation. As an advanced centre for substance metabolism and visceral regulation, the hippocampus is crucial for regulating visceral function. The liver is the core organ of material metabolism, and its afferent signals are mainly projected to the nucleus of the solitary tract (NTS) through vagus nerve, and then they are projected to the hypothalamus and limbic system.

Materials and methods: We placed a stereotaxic instrument on the head of each rat and performed craniotomy to open a window above the left hippocampus. We used gold-plated tungsten electrodes to monitor hippocampal neuronal discharges. Grounding was achieved using screws and silver wire. We electrically stimulated the liver branch of the vagus nerve and observed changes in hippocampal neuron discharges using a biological method; in this way, we identified hepatosensitive hippocampal region. We injected FluoroGold into this region and related brain areas. After 3 days, the rats were sacrificed and perfused; the hippocampi were fixed, dehydated, frozen, sectioned, and subjected to fluorescence microscopy. Results: Nerve discharge frequency and amplitude significantly increased in the hippocampal CA3 region (AP: -4.9, ML: -5.1, DV: $-5.0 \mathrm{~mm}$ ). After FluoroGold was injected into the left hepatosensitive region in the hippocampus, labelled cells were found in the contralateral hippocampus, ipsilateral piriform cortex $(P C)$, locus coeruleus (LC) and bilateral lateral hypothalamus (LHA); fluorescence in the ipsilateral hypothalamus was stronger than that of the contralateral hypothalamus. FluoroGold was injected into the $L H A, P C$, and $L C$; no labelled cells were found in the hippocampal CA3 region or in the control group.

Conclusions: The hippocampal CA3 area of rats may contain a hepatosensitive region that plays important roles in the regulation of liver and other organ function. This region may receive input from the LHA, PC, and LC. (Folia Morphol 2022; 81, 2: 261-270)

Key words: neural regulation of liver function, hepatic branch of the vagus nerve, hippocampus, neural pathways

Address for correspondence: Dr. C. Xia, Department of Human Anatomy, Basic Medical College of Guilin Medical University, China, e-mail: xiachunbo910@163.com

${ }^{*}$ Co-first author

This article is available in open access under Creative Common Attribution-Non-Commercial-No Derivatives 4.0 International (CC BY-NC-ND 4.0) license, allowing to download articles and share them with others as long as they credit the authors and the publisher, but without permission to change them in any way or use them commercially. 


\section{INTRODUCTION}

The hippocampus plays a crucial role in visceral regulation. Recent works on limbic system connections in the human brain have revealed a close relationship between that system and visceral information [5]. The hippocampus affects visceral function by regulating the excitability of the hypothalamic, autonomic nerve centre. The liver is the core metabolic organ; afferent signals project principally to the nucleus of the solitary tract (NTS) of the visceral centre, via the vagus nerve, and then from the nucleus to the hypothalamus and limbic system [10]. Neuroanatomical studies [8] have revealed that visceral sensory feedback signals reach the hypothalamus and the limbic brain, which controls food intake. Anatomically, direct neural projections from the hippocampus to the lateral hypothalamus and bisynaptic connections from the hippocampus to other known hypothalamic sites involved in feeding control have been identified [6, 7]. The hippocampus is key in terms of integrating peripheral signals with other sensory information. Vagus nerve stimulation triggers the typical hippocampal evoked potentials. Electrophysiologically, various afferent nerves of hepatic branch of the vagus nerve respond to slight changes in glucose concentration, osmotic pressure, and portal circulation temperature. These afferent systems may thus contribute to blood sugar, extracellular osmotic pressure, and body temperature homeostasis. Normal liver function involves the central nervous system; the marked dependence of the liver on the brain, and the neuromodulatory complexity of liver function, may be underestimated. Sensory signals from the liver travel to many areas of the central nervous system, including the hippocampus and hypothalamus, which regulate autonomic and endocrine homeostasis. However, little is known about the mechanisms involved. The hippocampal-hepatic neural pathway has not been studied. As a first step, we identified a hepatosensitive region in the hippocampus and explored the hippocampal-liver neural pathway via nerve tracing. Hence, our results set the experimental foundation for in-depth research on how the nervous system regulates liver function.

\section{MATERIALS AND METHODS}

\section{Experimental animals}

Male Sprague-Dawley rats aged 6-8 weeks were purchased from the laboratory animal centre of Guilin Medical College (certificate \#SCXK GUI 2007-0001).
Ethical Approval number is GLMC201703022. FluoroGold (FG) was from KeyGen Biotech (Jiangsu, China, cat. no. kgmp023/kgmp023-1). We used 190 rats divided into two groups that underwent electrical stimulation of the hepatic branch of the vagus nerve (30 rats), or neural tracing (160 rats). FG was injected into the hepatosensitive area of the left hippocampus, the ipsilateral piriform cortex (PC), the locus coeruleus (LC), and the bilateral lateral hypothalamus (LHA) $(0.1,0.3$, and $0.5 \mu \mathrm{L}$, respectively) and the rats were divided into three subgroups of 10 rats. Each control subgroup (10 rats) was injected with normal saline. During preliminary electrical stimulation of the hepatic branch of the vagus nerve, we recorded hippocampal neuron discharges using gilded tungsten wire (KEDOUBC, Suzhou, China) as the recording electrode. We delivered stimuli of 3, 6, and $9 \mathrm{~V}$ to fasted rats (water was allowed). Animals were allowed to acclimate for at least 1 week before experimentation. Animal suffering and death were minimised, in line with the regulations of the People's Republic of China that address the use of experimental animals.

\section{Experimental methods}

\section{Electrical stimulation of the hepatic branch of the vagus nerve}

After intraperitoneal injection of $1 \%(\mathrm{w} / \mathrm{v})$ pentobarbital sodium in saline $(40 \mathrm{mg} / \mathrm{kg})$, rats under anaesthesia were positioned on a stereotaxic instrument and the scalp was incised along the median sagittal line using the fontanelle as the origin. With reference to a brain stereotaxic atlas, the hippocampal skull projection area was marked. Brain tissue was exposed by drilling a hole about $0.8 \mathrm{~mm}$ in diameter, followed by placement of a grounding screw of depth about $0.7 \mathrm{~mm}, 12 \mathrm{~mm}$ from the recording electrode. A gold-plated tungsten electrode (The exposed tip is 5-10 microns in diameter, impedance 1-2 M $\Omega$ ) was fixed to the instrument holder and adjusted to the required coordinates. We confirmed that all electrodes were well-insulated; only the tips conducted electricity. The belly was disinfected and laparotomy was performed to expose the liver and oesophagus. A saline swab was used to move the left lobe to the right of the oesophagus, thus completely exposing the oesophagus; then the vagus nerve of the oesophagus and the hepatic vagus nerve of the porta hepatis were visible. We placed the electrodes of an in-house vagus nerve stimulator (a peripherally insulated bare silver wire that touched only the liver branch of the 
nerve) about $1 \mathrm{~cm}$ apart, close to and distant from the centre of the liver branch of the vagus nerve; both electrodes were wound around the nerve (1.5 loops), the negative pole was placed at the proximal end of the nerve and the positive pole was placed at the distal end. We were careful to minimise tension to prevent nerve damage. The stimulatory electrodes were fixed to the abdominal wall and the front abdominal wall muscle to prevent slippage. The stimulatory electrode and recording electrodes were connected to the signal acquisition system (gain: $5 \mathrm{mV}$, time constant: $0.1 \mathrm{~s}$, filtering: $10000 \mathrm{~Hz}$ ) and the grounding wire was wound tightly around a screw and slowly passed into the hippocampal region. When the signal-to-noise ratio became greater than 3:1, we recorded neuronal discharges. We implanted the recording electrodes into different hippocampal areas and structures, and then restimulated the hepatic branch of the vagus nerve; the threshold intensity is the minimum output voltage that can increase or decrease the discharge amplitude and frequency of hippocampal neurons by $20 \%$. Amplitude and frequency changes $>20 \%$ were considered to be indicative of hepatosensitive region. As a control, saline solution containing tetrodotoxin (TTX: $2.5 \mathrm{ng} / 0.1 \mu \mathrm{L}$ ) was injected into the liver sensitive region in the hippocampus, and then vagus nerve was stimulated to observe the changes of neuron discharge in the liver sensitive area of hippocampus. Procedures for local microinjections have been described in detail elsewhere [24]. The signals were $1 \mathrm{~ms}$ in duration after $100 \mathrm{~ms}$ of delay, and 3,6 , or $9 \mathrm{~V}$ in intensity. Intermittent stimulation featured single coarse-voltage stimuli. Only stable discharges with signal-to-noise ratio $>3$ were recorded.

\section{FluoroGold nerve tracer}

The rats' skulls were drilled as described above and $3 \%(\mathrm{w} / \mathrm{v}) \mathrm{FG}(0.1,0.3$, or $0.5 \mu \mathrm{L})$ was subdurally injected into hepatosensitive region (AP: -4.9, ML: -5.1 , DV: $-5.0 \mathrm{~mm}$ ), the left HLA (AP: $-4.8, \mathrm{ML}:-1.0$, DV: $-8.1 \mathrm{~mm}$ ), the PC (AP: $-4.8, \mathrm{ML}:-7.5, \mathrm{DV}:-8.5$ $\mathrm{mm}$ ), and the LC (AP: $-9.16, \mathrm{ML}:-1.0, \mathrm{DV}:-6.5 \mathrm{~mm}$ ). Control rats received the same amounts of normal saline. The needle remained in place for $10 \mathrm{~min}$ after injection and the rats were held for $72 \mathrm{~h}$. After sacrifice via re-anaesthesia, the chest cavity was opened and the heart was exposed. An in-house blunt needle was used to enter the ascending aorta from the apex of the heart, and fixed with a needle holder to prevent slipping. The right auricle was removed and successively perfused with phosphate buffered saline (PBS) followed by $4 \%(\mathrm{v} / \mathrm{v})$ paraformaldehyde, first rapidly and then slowly. The brains were collected, fixed in $4 \%(\mathrm{v} / \mathrm{v})$ paraformaldehyde at $4^{\circ} \mathrm{C}$ for at least $24 \mathrm{~h}$; and successively placed in 10\%, 15\%, and $30 \%$ sucrose solutions at $4^{\circ} \mathrm{C}$ until the tissue sank to the bottom. The samples were dehydrated, frozen, and sectioned (15-20 $\mu \mathrm{m})$, and then the sections were placed on adhesive slides, which were sealed after washing with PBS. Finally, the samples were observed under a fluorescence microscope. FG was excited using ultraviolet light (irradiation $418 \mathrm{~nm}$, excitation $331 \mathrm{~nm}$ ). All operations proceeded in the dark. Labelled cells were counted. Sections of the neuronal cell body and axon served as retrograde markers when counting cells. For each case, five typical sections were selected and labelled cells were counted at $40 x$ and $200 \times$.

\section{Statistical analysis}

Discharge frequencies and amplitudes and FG-labelled cell counts using SPSS ver. 25.0 software. Data are given as arithmetic means with standard deviations. The paired t-test was used to compare discharges before and after stimulation. Comparison of groups with different electrical stimulation intensity and different $F G$ injection doses were evaluated via one-way ANOVA. The independent samples t-test was used to compare differences in the bilateral LHA; a $p$-value $<0.05$ was taken to indicate statistical significance.

\section{RESULTS}

\section{Identification of hepatosensitive region in the hippocampus}

The hepatic branches of the vagus nerve of 30 rats were electrically stimulated and hippocampal discharge frequencies and amplitudes were recorded. Most rats (26/30) exhibited increased (> 20\%) discharge frequencies and amplitudes from the hippocampal CA3 region (AP:-4.9, ML:-5.1, DV:-5.0 mm). The basic discharge frequency was $124.31 \pm 16.31 \mathrm{~Hz}$ and the amplitude was $90.77 \pm 14.23 \mu \mathrm{V}$; stimulation with 3,6 , and $9 \mathrm{~V}$ yielded values of $157.81 \pm 16.94 \mathrm{~Hz}$ and $180.81 \pm 10.50 \mu \mathrm{V}, 187.77 \pm 10.66 \mathrm{~Hz}$ and $264.88 \pm 10.94 \mu \mathrm{V}$, and $193.31 \pm 13.08 \mathrm{~Hz}$ and $265.23 \pm 12.91 \mu \mathrm{V}$, respectively (all $p<0.05$, ANOVA). Thus, both the frequency and amplitude of discharge increased as the stimulation increased (Fig. 1E, F), and 

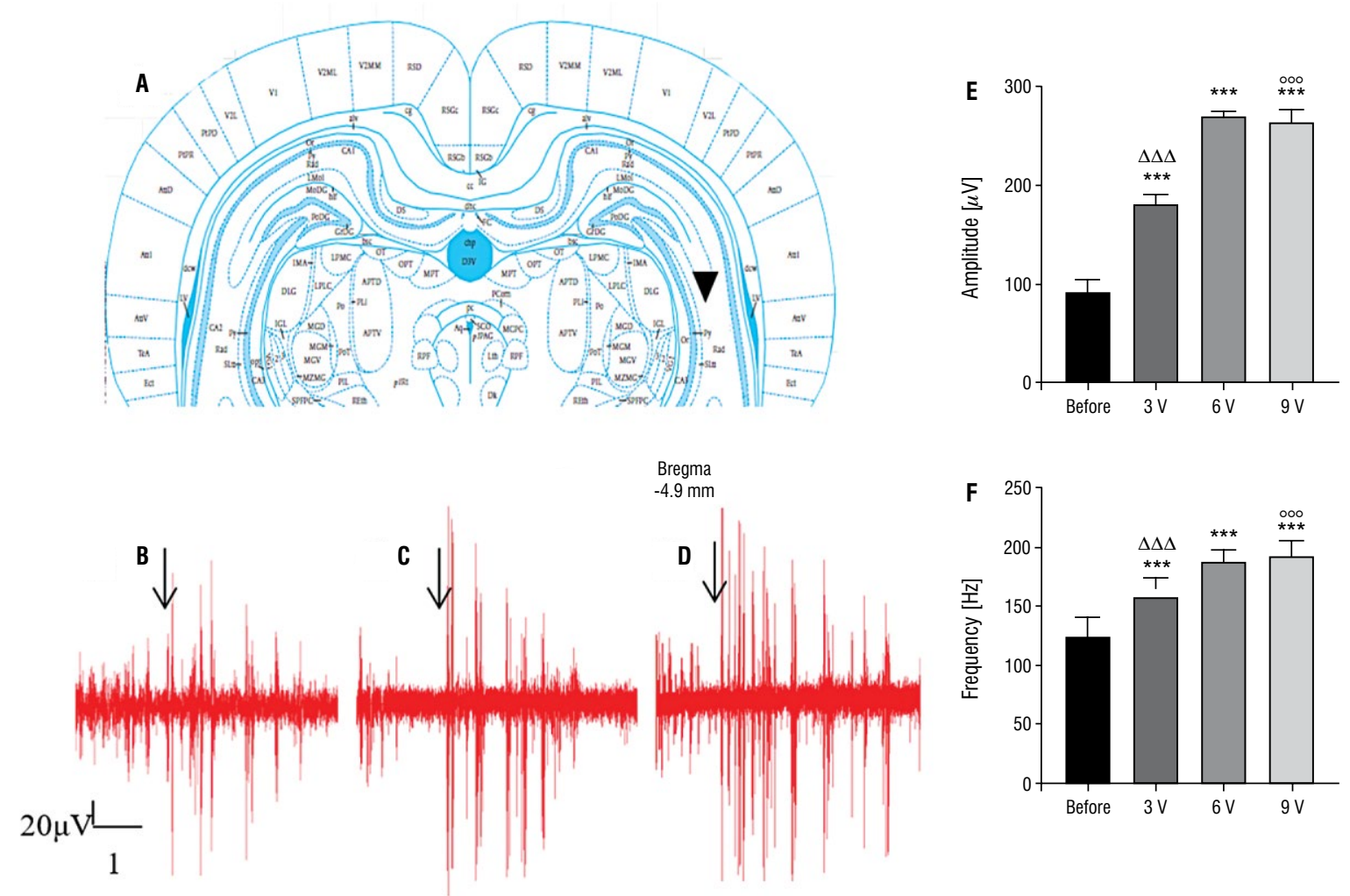

Figure 1. A. Schematic diagram of the location of recorded and microinjection in the hippocampus. Record and injections sites are indicated by inverted triangles $(\mathbf{\nabla})$; B-D. Variations of neuron discharge in rat hippocampal CA3 region of vagus nerve with different electrical stimulation $(\mathrm{n}=26)$; B. 3 V; C. 6 V; D. 9 V; $\downarrow$ : electrical stimulation; E, F. Rats hippocampal neuronal discharge frequency and amplitude $(n=26)$ with different intensity of liver branch of the vagus nerve stimulation; $\mathbf{E}$. The firing frequency of hippocampal region before stimulation and after stimulation of vagus nerve with different intensities; F. The discharge amplitude of hippocampal region after basal discharge before stimulation and hepatic branch of vagus nerve after electrical stimulation with different intensity. Data are processed with paired test and single factor variance analysis. ${ }^{* *} p<0.001$ - comparison before and after stimulus with different intensity; $\Delta \Delta \Delta p<0.001$ - comparison of results stimulated with $3 \mathrm{~V}$ and $6 \mathrm{~V} ; \circ \circ \circ \mathrm{p}<0.001$ - comparison of results stimulated with $3 \mathrm{~V}$ and $9 \mathrm{~V}$.

basically reached the saturation stimulation intensity at $6 \mathrm{~V}$, while the discharge changes of $6 \mathrm{~V}$ and $9 \mathrm{~V}$ in the liver sensitive areas of hippocampus showed no significant change. After stimulation ceased, the frequency and amplitude gradually returned to the basic level. As stimulus intensity increased, the recovery time was prolonged (Fig. 1B-D), After TTX injection, the local hippocampal action potential was blocked, eliminating the response to the stimulation of the hepatic branch of the vagus nerve.

Figure $1 \mathrm{~A}$ shows the location of the injection and record site in hippocampus. Though there were variations in the placements among animals, the histology indicated that electrode implantation and TTX were injected into the hepatic sensitive regions of the hippocampus of the rats used in the study. Rats with inaccurate localisation were excluded.

\section{Conduction pathways of hepatosensitive region in the hippocampus}

FluoroGold was injected into hepatosensitive region in the left hippocampus to explore the nerve conduction pathways. Haematoxylin and eosin staining and fluorescence microscopy of defined areas of the left hippocampus (excluding instances of injection inaccuracy or FG overflow) revealed various numbers of labelled cells. Different amounts of FG were injected into hepatosensitive areas of the left hippocampus, in the right hippocampus containing the FG labelled neurons, including both LHAs and the left sides of the PC and LC were seen with fluorescence labelling cells (Figs. 2-5). Labelled cell numbers significantly increased as the FG dose increased ( $p<0.05$; Table 1). The left lateral hypothalamus exhibited more labelled cells than the right side ( $p<0.05 ;$ Fig. 3M). After 

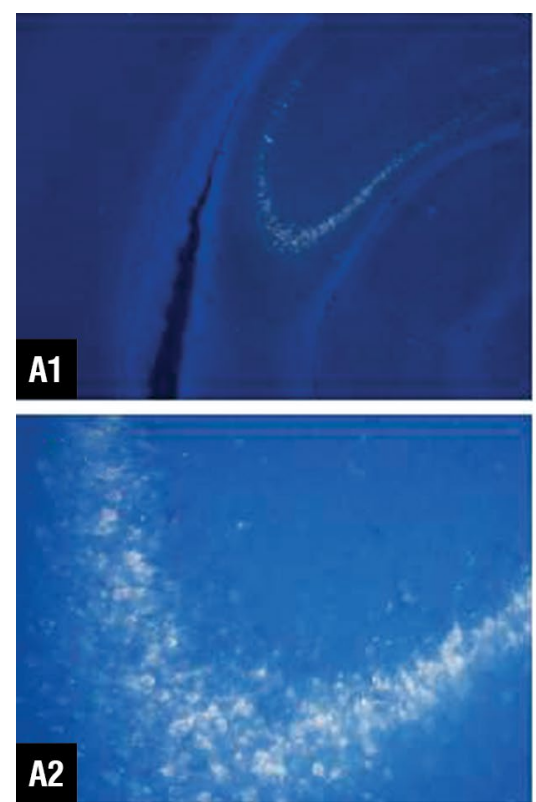
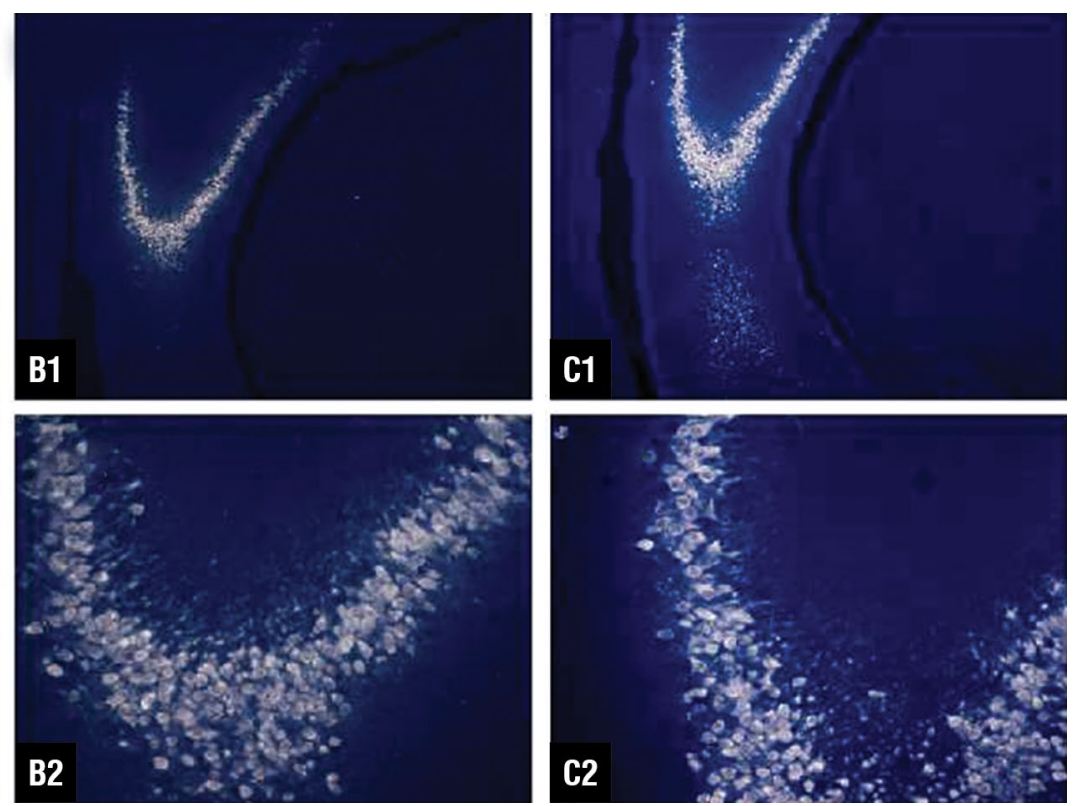

Figure 2. Fluorescence tracer results of right hippocampus after different doses of FluoroGold $(F G)$ were injected into left hippocampal liver sensitive region of rats $(0.1 \mu \mathrm{L}, \mathrm{n}=10 ; 0.3 \mu \mathrm{L}, \mathrm{n}=10 ; 0.5 \mu \mathrm{L}, \mathrm{n}=10 ; \mathbf{A 1}$. FG $0.1 \mu \mathrm{L}, 40 \times, \mathbf{A 2}$. FG $0.1 \mu \mathrm{L}, 200 \times$; B1. FG $0.3 \mu \mathrm{L}, 40 \times$; B2. FG $0.3 \mu \mathrm{L}, 200 \times$; C1. FG $0.5 \mu \mathrm{L}, 40 \times$; C2. FG $0.5 \mu \mathrm{L}, 200 \times$ ). The bright white neurons are FG labelled cells.

saline injection, no labelled cells were found in the right hippocampus, the bilateral LHAs, the PC, or LC. After FG was injected into the LHA, PC, and LC, no labelled cells were found in the hippocampus (Fig. 6).

\section{DISCUSSION}

The hippocampus plays an important role in visceral regulation [2]; research on this topic is of both theoretical and clinical significance. The liver sends signals to the central nervous system through the vagus nerve [31]. We electrically stimulated the liver branch of the vagus nerve and found hepatosensitive region in the hippocampus. The method used was developed in 1986 by Kobashi and Adachi [15], who studied the projections of related nuclei. Other studies featuring electrical stimulation of the liver branch of the vagus nerve have revealed a functional relationship between the liver and the nucleus tractus solitarius (NTS). We found that, in the hippocampal CA3 region (AP: $-4.9, \mathrm{ML}:-5.1, \mathrm{DV}:-5.0 \mathrm{~mm}$ ), the discharge frequencies and amplitudes increased by $>20 \%$, and then gradually reverted to the basic rates, consistent with the findings of previous studies [17]. We speculate that the increased discharges in specific hippocampal areas caused by stimulation of the hepatic branch of the vagus nerve may reflect projections of the hippocampal liver region [32]. In a previous study, stimulation of the hepatic branch of the vagus nerve reduced weight gain in rats fed a high-fat diet [20]. Such stimulation also triggers loss of weight and appetite in other animals; the vagus nerve transmits signals to both the hippocampus and other brain regions [23]. In young mice, metabolic data on the liver are transmitted to the brain via the hepatic vagus nerve $[3,28-30]$. These works, combined with our present data, suggest that hepatic afferent signals may be projected to specific hippocampal areas by the hepatic branch of the vagus nerve. We found that the hippocampus (AP: -4.9 , ML: $-5.1, \mathrm{DV}:-5.0 \mathrm{~mm}$ ) may be sensitive to signals from the liver vagus nerve, playing an important role in the regulation of liver and other organ function. Different neurons exhibit different action potential thresholds. For example, intermediate hippocampal neurons (inhibitory neurons) have lower thresholds than pyramidal cells (primary neurons). That cone cell discharges might be inhibited by local intermediate neurons activated by certain stimuli delivered to their afferent fibres. Although computational modelling supports this hypothesis [21], in vivo data are lacking. In 1 study [9], neuronal stimulation had both inhibitory and excitatory effects. In addition, the stimulation frequency may affect neuronal discharge [25]. We used 3, 6, and $9 \vee$ to stimulate the hepatic branch of the vagus nerve. The neuronal discharge gradually increased as stimulation intensity increased, perhaps 

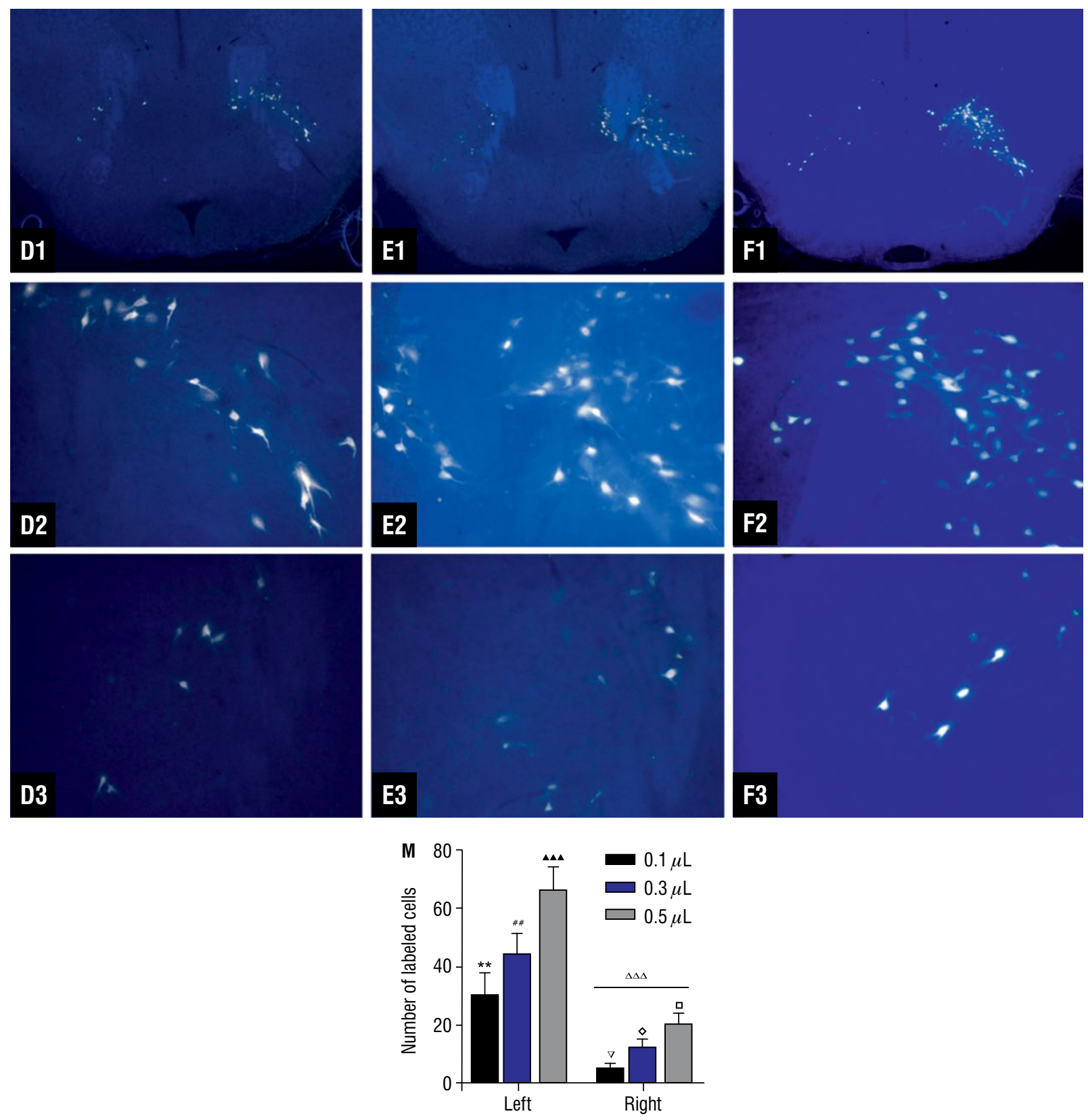

Figure 3. D-F. Fluorescence tracer results of bilateral lateral hypothalamus after different doses of FluoroGold (FG) were injected into left hippocampal liver sensitive region of rats $(0.1 \mu \mathrm{L}, \mathrm{n}=10 ; 0.3 \mu \mathrm{L}, \mathrm{n}=10 ; 0.5 \mu \mathrm{L}, \mathrm{n}=10 ; \mathbf{D} 1$. FG $0.1 \mu \mathrm{L}, 40 \times$; D2. FG $0.1 \mu \mathrm{L}$, left side $200 \times$; D3. FG $0.1 \mu \mathrm{L}$, right side 200×; E1. FG $0.3 \mu \mathrm{L}, 40 \times$; E2. FG $0.3 \mu \mathrm{L}$, left side 200×; E3. FG $0.3 \mu \mathrm{L}$, right side 200×; F1. FG $0.5 \mu \mathrm{L}, 40 \times$; F2. FG $0.5 \mu \mathrm{L}$, left side $200 \times$; F3. FG $0.5 \mu \mathrm{L}$, right side $200 \times$ ). The bright white neurons are FG labelled cells; M. Number of fluorescencelabelled cells in the left and right lateral hypothalamus of the hippocampal hepatosensitive region injected with different doses of $F G(0.1 \mu \mathrm{L}$, $\mathrm{n}=10 ; 0.3 \mu \mathrm{L}, \mathrm{n}=10 ; 0.5 \mu \mathrm{L}, \mathrm{n}=10$ ). One-way ANOVA and independent sample $\mathrm{t}$ test are used; $\Delta \Delta \Delta \mathrm{p}<0.001$ - compare lateral hypothalamus (LHA) of left and right at the same dose; ${ }^{* *} p 0.01$ left LHA: $0.1 \mu \mathrm{L}$ vs. $0.3 \mu \mathrm{L} ; \# \# \mathrm{p}<0.01-0.3 \mu \mathrm{L}$ vs. $0.5 \mu \mathrm{L}$;

$\Delta \Delta \Delta \mathrm{p}<0.001-0.1 \mu \mathrm{L}$ vs. $0.5 \mu \mathrm{L}$; Right LHA: $\nabla \mathrm{p}<0.05-0.1 \mu \mathrm{L}$ vs. $0.3 \mu \mathrm{L} ;$ op $<0.05-0.3 \mu \mathrm{L}$ vs. $0.5 \mu \mathrm{L} ; \square \mathrm{p}<0.05-0.1 \mu \mathrm{L}$ vs. $0.5 \mu \mathrm{L}$.

attributable to the stimulation of pyramidal cells. The neuronal discharge frequency and amplitude gradually increased with stimulation intensity, perhaps reflecting increasing pyramidal cell excitation. Changes in hippocampal neuronal discharge developed within
$0.1 \mathrm{~s}$ after electrical stimulation of the vagus nerve; the neuroregulatory effects were rapid, accurate, and transient. Compared to injection of glucose or lipids into the hepatic portal vein, nerve stimulation detects sensitive brain areas more rapidly and ac- 

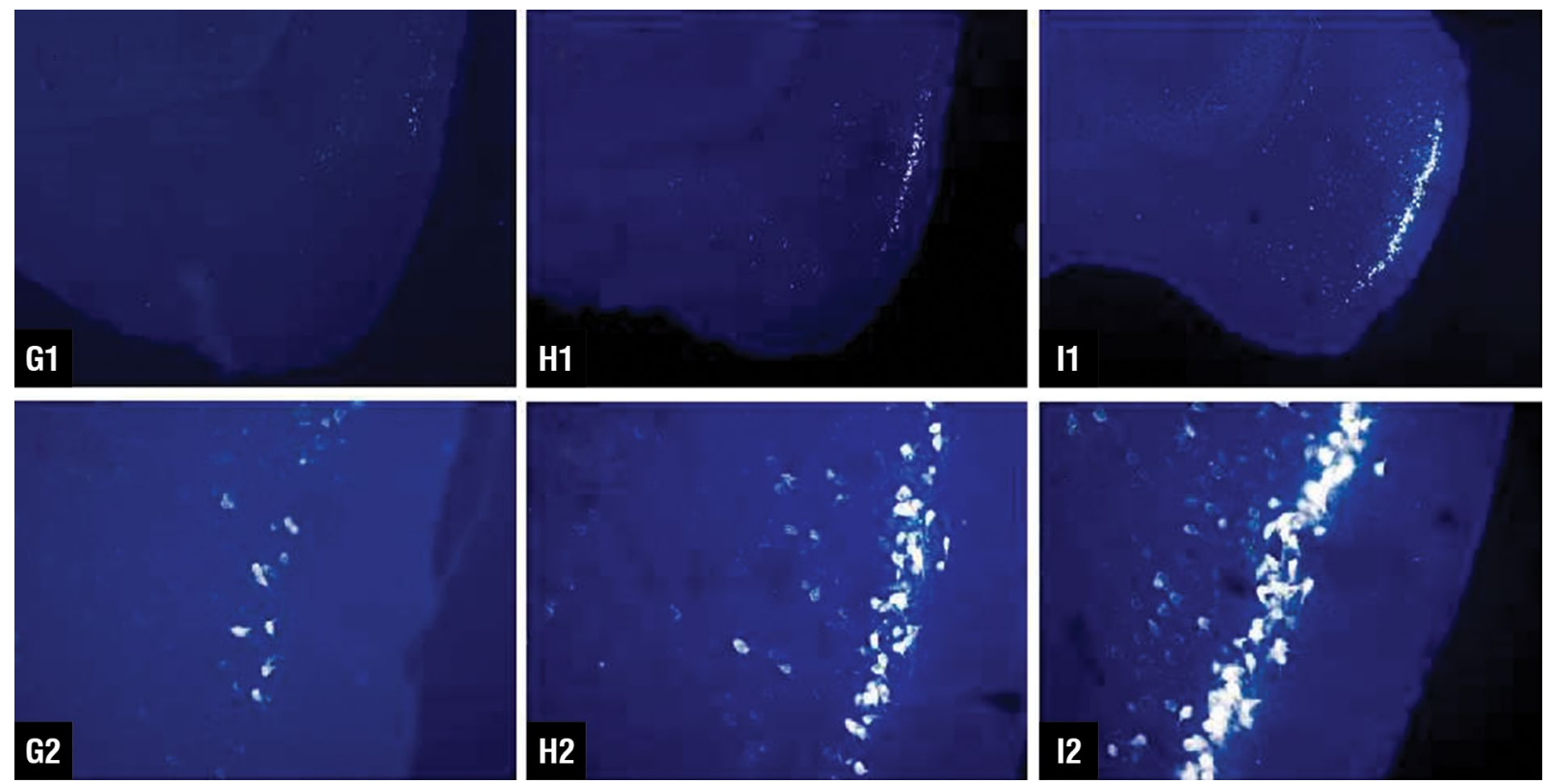

Figure 4. Fluorescence tracing results of ipsilateral pyriform cortex after different doses of FluoroGold (FG) were injected into left hippocampal liver sensitive region of rats $(0.1 \mu \mathrm{L}, \mathrm{n}=10 ; 0.3 \mu \mathrm{L}, \mathrm{n}=10 ; 0.5 \mu \mathrm{L}, \mathrm{n}=10 ; \mathbf{G 1}$. FG $0.1 \mu \mathrm{L}, 40 \times$; G2. FG $0.1 \mu \mathrm{L}, 200 \times$; H1. FG $0.3 \mu \mathrm{L}, 40 \times$; H2. FG $0.3 \mu \mathrm{L}, 200 \times$; I1. FG $0.5 \mu \mathrm{L}, 40 \times$; I2. FG $0.5 \mu \mathrm{L}, 200 \times$ ). The bright white neurons are FG labelled cells.
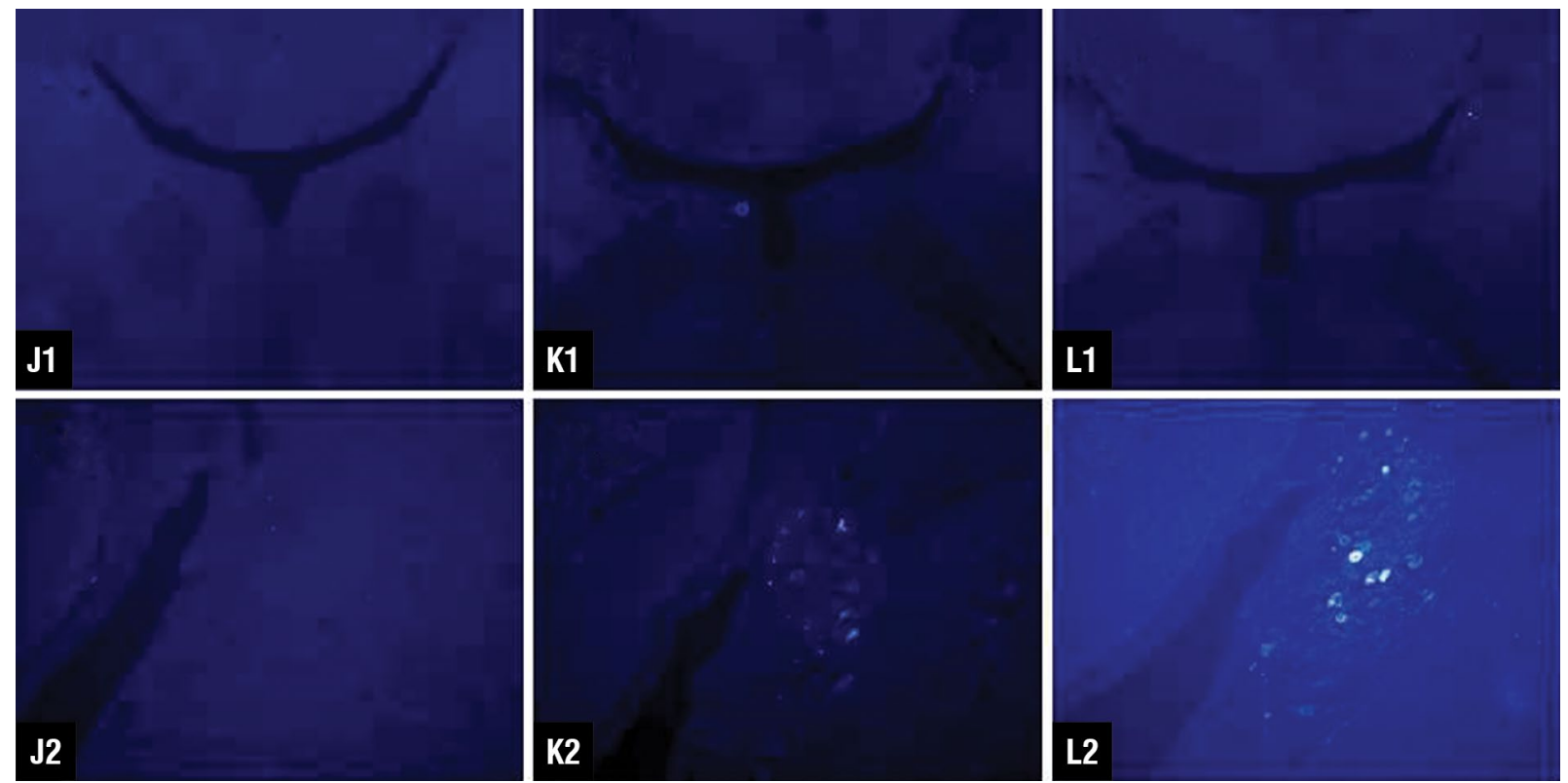

Figure 5. Fluorescence tracing results of ipsilateral locus coeruleus after different doses of FluoroGold (FG) were injected into left hippocampal liver sensitive region of rats $(0.1 \mu \mathrm{L}, \mathrm{n}=10 ; 0.3 \mu \mathrm{L}, \mathrm{n}=10 ; 0.5 \mu \mathrm{L}, \mathrm{n}=10 ; \mathbf{J 1}$. FG $0.1 \mu \mathrm{L}, 40 \times ; \mathbf{J 2}$. FG $0.1 \mu \mathrm{L}, 200 \times ; \mathbf{K 1}$. FG $0.3 \mu \mathrm{L}$, 40×; K2. FG $0.3 \mu \mathrm{L}, 200 \times$; L1. FG $0.5 \mu \mathrm{L}, 40 \times$; L2. FG $0.5 \mu \mathrm{L}, 200 \times$ ). The bright white neurons are FG labelled cells.

curately. It is possible that the observed changes in nerve discharges reflect only signals transmitted via the hepatic branch of the vagus nerve. It is also possible that stimulation of this branch affects not only the liver but also the gastrointestinal tract [1]. Given the unique features of the nerve, the affected brain regions become apparent upon neuronal stimulation. However, associations among vagus nerve-sensitive regions remain poorly understood. The nerve exhibits many and varied functions that may overlap; roles 
Table 1. Numbers of fluorescently labelled cells in the right hippocampus, bilateral lateral hypothalamus (LHA), left piriform cortex $(\mathrm{PC})$, and locus coeruleus (LC) after hepatosensitive region in the left hippocampus were injected with different doses of FluoroGold

\begin{tabular}{lcccccc}
\hline Group & Samples & Right hippocampus & Left LHA & Right LHA & Left PC & Left LC \\
\hline $0.1 \mu \mathrm{L}$ & 10 & $50.63 \pm 5.04$ & $30.50 \pm 7.39$ & $5.25 \pm 1.67$ & $21.38 \pm 5.71$ & $10.25 \pm 3.24$ \\
$0.3 \mu \mathrm{L}$ & 10 & $136.00 \pm 9.64$ & $44.43 \pm 7.00$ & $12.71 \pm 2.63$ & $56.43 \pm 9.88$ & $19.14 \pm 2.41$ \\
$0.5 \mu \mathrm{L}$ & 10 & $267.67 \pm 16.92$ & $66.44 \pm 7.99$ & $20.67 \pm 3.39$ & $115.33 \pm 8.34$ & $25.11 \pm 5.06$ \\
F-value & & 707.33 & 49.59 & 69.28 & 294.85 & 31.53 \\
P-value & & $<0.05$ & $<0.05$ & $<0.05$ & $<0.05$ & $<0.05$ \\
\hline
\end{tabular}
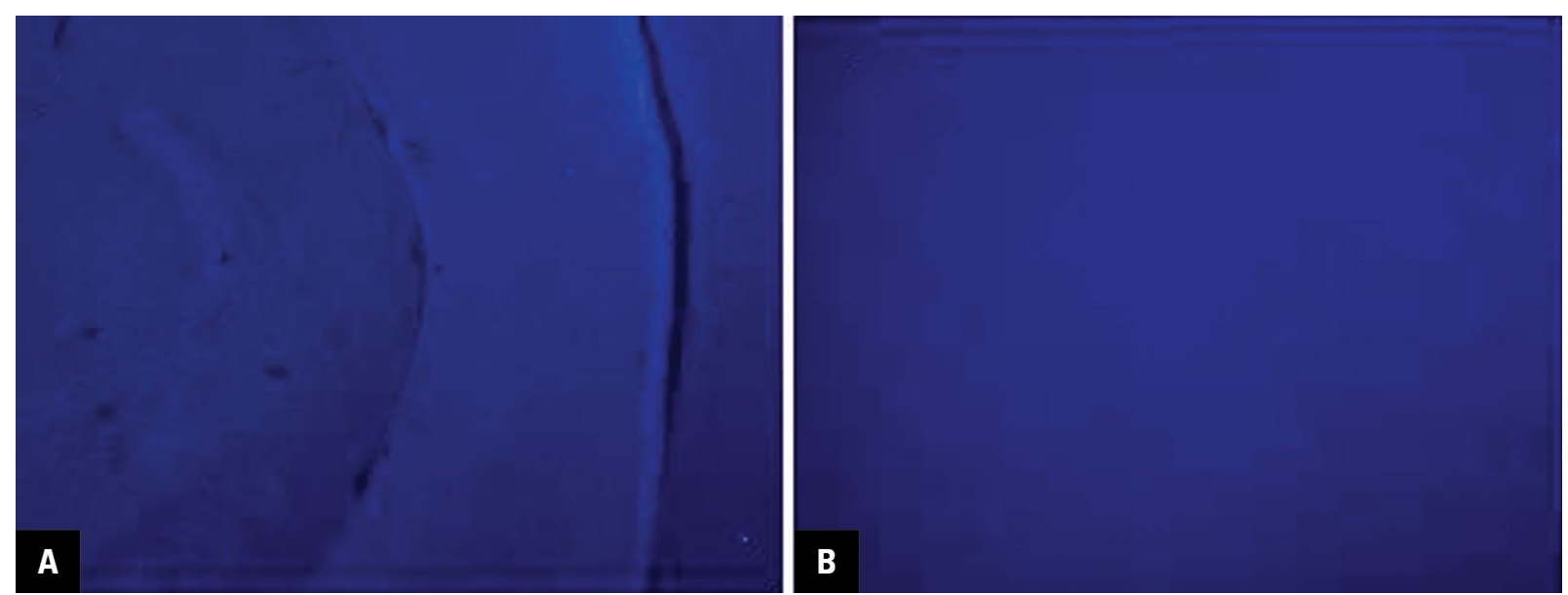

Figure 6. Fluorescence labelled results of FluoroGold injection in the left lateral hypothalamus, pyriform cortex and locus coeruleus in rats $(n=10)$. There are not fluorescent labelled cells in the left hippocampal liver sensitive area; A. $0.5 \mu \mathrm{L}, 40 \times$; B. $0.5 \mu \mathrm{L}, 200 \times$.

may be exchanged. Thus, it remains to be confirmed that stimulation of the vagus nerve of the liver triggers the hippocampal discharges. Despite the several neuroanatomical possibilities, we have confirmed a connection between the hippocampus (the visceral regulatory centre) and the liver. The BL-420E experimental instrument used in this experiment may have some limitations, and the specific mechanism of the hippocampal hepatic sensitive nerve area and its related neural pathways still needs to be further studied.

We used FG (a retrograde nerve tracer) to explore the morphological and anatomical connections of conduction pathways in hepatosensitive areas of the hippocampus. FG reveals fibres that run from the brain to target organs, and is the gold standard tracer for rodents. We injected it into hepatosensitive areas in the left hippocampus; labelled cells were apparent in the right hippocampus, bilateral LHA, left PC and LC, and ipsilateral LHA, at levels much higher than in the contralateral regions. Recent studies have shown that the LHA is involved in the regulation of gastrointestinal movement and feeding behaviour via the brainstem NTS and the dorsal vagus motor nucleus. Thus, the hypothalamus is involved in visceral regulation. Previous studies have found that PC fibres project into the hypothalamus and amygdala and regulate appetite. The LC-norepinephrine (NE) system mediates many visceral responses and is associated with hippocampal fibres. We have shown that fibrous connections may exist between the left and right hippocampi. Both LHAs, and the ipsilateral PC and LC, project fibres to the hepatosensitive area of the hippocampus; the ipsilateral LHA projection is stronger than the contralateral projection. One side of the hepatosensitive area of the hippocampus accepts fibres projecting from the bilateral hypothalamus; this tissue centrally regulates energy and metabolic balance [27] including liver lipid and lipoprotein metabolism. Hypothalamic fibres project to the hippocampus. Orexin is found in both the hippocampus and LC; both tissues regulate feeding behaviour and energy balance. Our results are consistent with those of recent studies on the anatomy of the association between the hippocampus, hypothalamus, and LC 
$[11,14]$. We speculate that liver function regulation by hepatosensitive region in the hippocampal CA3 region may involve the $\mathrm{LHA}, \mathrm{PC}$, and $\mathrm{LC}$. When FG was injected into these regions, no labelled cells were found in the hepatosensitive areas, suggesting that the fibre connections between the hepatosensitive region and the LHA, PC, and LC may be one-way [19]. Some recent studies $[4,12,22]$ found that the hippocampus was directly or indirectly linked to the hypothalamus, PC, and LC. The hippocampal control hierarchy [16] is: hippocampus to the hypothalamus, to the brain stem, to the vagus nerve. Functional fibres linking the hippocampus and hypothalamus have been found in previous studies [26]. The responses in the hippocampus to vagal stimulation are likely to result from stimulation of vagal afferents and reach the hippocampus via NTS and the data do not test whether there is a vagal efferent pathway involved. A recent study found that the PC and hippocampus were involved in the sense of smell [13]. Furthermore, the hippocampus and LC affect cognitive function [18]. However, few studies have explored whether the $\mathrm{PC}$ and $\mathrm{LC}$ regulate internal organs. The hepatosensitive hippocampal CA3 region may be connected to the lateral hypothalamus, $\mathrm{PC}$, and LC. The roles played by the latter two regions will be explored in future experiments.

\section{CONCLUSIONS}

There may be liver sensitive region in the CA3 area of rat hippocampus, and the area receives nerve fibre projections from lateral hypothalamic area, piriform cortex and blue spots, and is unidirectional fibre projection. The CA3 area received nerve fibre projection from bilateral lateral hypothalamus area, and the ipsilateral projection was stronger than the contralateral side.

\section{Funding}

National Natural Science Foundation of China (81760159, 81960163); Guangxi Natural Science Foundation (2017GXNSFAA198307, 2018GXNSFBA138029); Open Project of Guangxi Key laboratory of Brain and Cognitive Neuroscience (gklbcn-20180105-05).

\section{Conflict of interest: None declared}

\section{REFERENCES}

1. Agster KL, Burwell RD. Hippocampal and subicular efferents and afferents of the perirhinal, postrhinal, and entorhinal cortices of the rat. Behav Brain Res. 2013;
254: 50-64, doi: 10.1016/j.bbr.2013.07.005, indexed in Pubmed: 23872326.

2. Arrigo A, Mormina E, Calamuneri A, et al. Amygdalar and hippocampal connections with brainstem and spinal cord: a diffusion MRI study in human brain. Neuroscience. 2017; 343: 346-354, doi: 10.1016/j. neuroscience.2016.12.016, indexed in Pubmed: 28003162.

3. Bernal-Mizrachi C, Xiaozhong L, Yin Li, et al. An afferent vagal nerve pathway links hepatic PPARalpha activation to glucocorticoid-induced insulin resistance and hypertension. Cell Metab. 2007; 5(2): 91-102, doi: 10.1016/j. cmet.2006.12.010, indexed in Pubmed: 17276352.

4. Blessing EM, Beissner F, Schumann A, et al. A data-driven approach to mapping cortical and subcortical intrinsic functional connectivity along the longitudinal hippocampal axis. Hum Brain Mapp. 2016; 37(2): 462-476, doi: 10.1002/hbm.23042, indexed in Pubmed: 26538342.

5. Catani M, Dell'acqua F, Thiebaut de Schotten M. A revised limbic system model for memory, emotion and behaviour. Neurosci Biobehav Rev. 2013; 37(8): 1724-1737, doi: 10.1016/j.neubiorev.2013.07.001, indexed in Pubmed: 23850593.

6. Cenquizca LA, Swanson LW. Analysis of direct hippocampal cortical field CA1 axonal projections to diencephalon in the rat. J Comp Neurol. 2006; 497(1): 101-114, doi: 10.1002/ cne.20985, indexed in Pubmed: 16680763.

7. Cenquizca LA, Swanson LW. Spatial organization of direct hippocampal field CA1 axonal projections to the rest of the cerebral cortex. Brain Res Rev. 2007; 56(1): 1-26, doi: 10.1016/j.brainresrev.2007.05.002, indexed in Pubmed: 17559940.

8. Davidson TL, Chan K, Jarrard LE, et al. Contributions of the hippocampus and medial prefrontal cortex to energy and body weight regulation. Hippocampus. 2009; 19(3): 235-252, doi: 10.1002/hipo.20499, indexed in Pubmed: 18831000.

9. Feng Z, Wang Z, Guo Z, et al. High frequency stimulation of afferent fibers generates asynchronous firing in the downstream neurons in hippocampus through partial block of axonal conduction. Brain Res. 2017; 1661: 67-78, doi: 10.1016/j.brainres.2017.02.008, indexed in Pubmed: 28213155.

10. Hannapel RC, Henderson YH, Nalloor R, et al. Ventral hippocampal neurons inhibit postprandial energy intake. Hippocampus. 2017; 27(3): 274-284, doi: 10.1002/ hipo.22692, indexed in Pubmed: 28121049.

11. Jennings JH, Rizzi G, Stamatakis AM, et al. The inhibitory circuit architecture of the lateral hypothalamus orchestrates feeding. Science. 2013; 341(6153): 1517-1521, doi: 10.1126/science.1241812, indexed in Pubmed: 24072922.

12. Jeon SG, Kim YJ, Kim KAh, et al. Visualization of altered hippocampal connectivity in an animal model of Alzheimer's disease. Mol Neurobiol. 2018; 55(10): 7886-7899, doi: 10.1007/s12035-018-0918-y, indexed in Pubmed: 29488134.

13. Jiang $H$, Schuele $S$, Rosenow J, et al. Theta oscillations rapidly convey odor-specific content in human piriform cortex. Neuron. 2017; 94(1): 207-219.e4, doi: 10.1016/j. neuron.2017.03.021, indexed in Pubmed: 28384472.

14. Kempadoo KA, Mosharov EV, Choi SeJ, et al. Dopamine release from the locus coeruleus to the dorsal hippocam- 
pus promotes spatial learning and memory. Proc Natl Acad Sci USA. 2016; 113(51): 14835-14840, doi: 10.1073/ pnas.1616515114, indexed in Pubmed: 27930324.

15. Kobashi M, Adachi A. Projection of nucleus tractus solitarius units influenced by hepatoportal afferent signal to parabrachial nucleus. J Auton Nerv Syst. 1986; 16(2): 153-158, doi: 10.1016/0165-1838(86)90006-8, indexed in Pubmed: 3722718.

16. Kobrzycka A, Napora P, Pearson BL, et al. Peripheral and central compensatory mechanisms for impaired vagus nerve function during peripheral immune activation. J Neuroinflammation. 2019; 16(1): 150, doi: 10.1186/ s12974-019-1544-y, indexed in Pubmed: 31324250.

17. Larsen LE, Wadman WJ, Marinazzo D, et al. Vagus nerve stimulation applied with a rapid cycle has more profound influence on hippocampal electrophysiology than a standard cycle. Neurotherapeutics. 2016; 13(3): 592-602, doi: 10.1007/s13311-016-0432-8, indexed in Pubmed: 27102987.

18. Lashgari R, Khakpour-Taleghani B, Motamedi F, et al. Effects of reversible inactivation of locus coeruleus on long-term potentiation in perforant path-DG synapses in rats. Neurobiol Learn Mem. 2008; 90(2): 309-316, doi: 10.1016/j.nlm.2008.05.012, indexed in Pubmed: 18577458.

19. Letzner S, Simon A, Güntürkün O. Connectivity and neurochemistry of the commissura anterior of the pigeon (Columba livia). J Comp Neurol. 2016; 524(2): 343-361, doi: 10.1002/cne.23858, indexed in Pubmed: 26179777.

20. López-Soldado I, Fuentes-Romero R, Duran J, et al. Effects of hepatic glycogen on food intake and glucose homeostasis are mediated by the vagus nerve in mice. Diabetologia. 2017; 60(6): 1076-1083, doi: 10.1007/s00125-017-4240-4, indexed in Pubmed: 28299379.

21. Mahmud M, Vassanelli S. Differential modulation of excitatory and inhibitory neurons during periodic stimulation. Front Neurosci. 2016; 10: 62, doi: 10.3389/ fnins.2016.00062, indexed in Pubmed: 26941602.

22. Manchanda S, Kaur G. Withania somnifera leaf alleviates cognitive dysfunction by enhancing hippocampal plasticity in high fat diet induced obesity model. BMC Complement Altern Med. 2017; 17(1): 136, doi: 10.1186/s12906-0171652-0, indexed in Pubmed: 28253924.

23. O'Leary OF, Ogbonnaya ES, Felice $D$, et al. The vagus nerve modulates BDNF expression and neurogenesis in the hippocampus. Eur Neuropsychopharmacol. 2018; 28(2): 307-316, doi: 10.1016/j.euroneuro.2017.12.004, indexed in Pubmed: 29426666.

24. Pereira de Vasconcelos A, Klur S, Muller C, et al. Reversible inactivation of the dorsal hippocampus by tetrodotoxin or lidocaine: a comparative study on cerebral functional activity and motor coordination in the rat. Neuroscience. 2006; 141(4): 1649-1663, doi: 10.1016/j.neuroscience.2006.05.023, indexed in Pubmed: 16797129.

25. Qiu C, Feng Z, Zheng L, et al. Selective modulation of neuronal firing by pulse stimulations with different frequencies in rat hippocampus. Biomed Eng Online. 2019; 18(1): 79, doi: 10.1186/s12938-019-0700-z, indexed in Pubmed: 31337402.

26. Reppucci CJ, Petrovich GD. Organization of connections between the amygdala, medial prefrontal cortex, and lateral hypothalamus: a single and double retrograde tracing study in rats. Brain Struct Funct. 2016; 221(6): 2937-2962, doi: 10.1007/s00429-015-1081-0, indexed in Pubmed: 26169110.

27. Taher J, Farr S, Adeli K. Central nervous system regulation of hepatic lipid and lipoprotein metabolism. Curr Opin Lipidol. 2017; 28(1): 32-38, doi: 10.1097/ MOL.0000000000000373, indexed in Pubmed: 27906714.

28. Tsukita S, Yamada T, Uno K, et al. Hepatic glucokinase modulates obesity predisposition by regulating BAT thermogenesis via neural signals. Cell Metab. 2012; 16(6): 825-832, doi: 10.1016/j.cmet.2012.11.006, indexed in Pubmed: 23217261.

29. Uno K, Katagiri H, Yamada T, et al. Neuronal pathway from the liver modulates energy expenditure and systemic insulin sensitivity. Science. 2006; 312(5780): 1656-1659, doi: 10.1126/science.1126010, indexed in Pubmed: 16778057.

30. Uno K, Yamada T, Ishigaki Y, et al. A hepatic amino acid/ mTOR/S6K-dependent signalling pathway modulates systemic lipid metabolism via neuronal signals. Nat Commun. 2015; 6: 7940, doi: 10.1038/ncomms8940, indexed in Pubmed: 26268630.

31. Yahagi N. Hepatic control of energy metabolism via the autonomic nervous system. J Atheroscler Thromb. 2017; 24(1): 14-18, doi: 10.5551/jat.RV16002, indexed in Pubmed: 27592630 .

32. Zanos TP, Silverman HA, Levy $T$, et al. Identification of cytokine-specific sensory neural signals by decoding murine vagus nerve activity. Proc Natl Acad Sci USA. 2018; 115(21): E4843-E4852, doi: 10.1073/pnas.1719083115, indexed in Pubmed: 29735654. 